of a slight alteration in the magnetic properties of clay produced by firing. The Signals Research and Development Establishment demonstrated a rubidium-vapour magnetometer, which is best described as the optical-pumping (or 'photon-spin pumping') device working in reverse. The effect of a magnetic field, which separates the otherwise degenerate spinlevels by an extent proportional to this field if it is small, is used to measure the strength of the field. A perpendicular radio-frequency magnetic field is adjusted to the energy difference between the pair of levels which have been population-inverted (that is, to the Larmor frequency when the system starts to operate maser-wise, a state that is detected by the strong absorption of the pumping beam of circularly polarized rubidium $D_{1}$ light). Thus, instead of tuning to a given radio-frequency by altering the field, the system is tuned to the ambient field by altering the frequency. This is sufficiently sensitive for geomagnetic observations, and may possibly be applied to aerial and space magnetic surveys; indeed, the actual apparatus was too sensitive to work in the disturbing fields of the Hall, and was planted out on the Westminster School playing-field.
These are the impressions of a day's visit; a proper appreciation would have needed the full five days, and some considerable preparation beforehand. There was much that I missed, and one highlight in the form of a miniature lighthouse with a brilliant source that I even failed to identify properly. The exhibits from various research laboratories of the Department of Scientific and Industrial Research were representative of the wide variety of tasks they undertake. The Science Museum had two exhibits, one ancient and one modern, and there were six contributions from university departments ; it would add further facets to a brilliant occasion if we could see, among all the new tools for research and development, more of the new apparatus designed at the universities for their practical classes and demonstrations, and at the Science Museum for the instruction of the general public.

Evening discourses were given during the Exhibition by F. S. Burt, on "Hydrodynamics Research"; by Dr. G. E. R. Deacon, on "The Physics of the Ocean"; and by Dr. F. P. Bowden, on "Some Physical Problems in Travelling at Supersonic Speed". G. R. NOAKES

\title{
CALORIE BALANCE IN MAN
}

T HE one hundred and thirty-seventh meeting of the Nutrition Society was held in the Middlesex Hospital Medical School on December 17, and took the form of a symposium on "Calorie Balance in Man". Perhaps it was optimistic to expect a symposium, continuing from 10.15 a.m. to 4.30 p.m. on the Satur. day a week before Christmas, to be popular. In fact, it was a well-attended meeting with an audience of more than 100. The chairman was Sir Charles Dodds.

Not so many years ago the subject of "Calorie Balance" would have been an unlikely topic for such a symposium. Interest in calories, at least in relation to human energy, was at a low ebb scientifically and a rather naive idea was prevalent that little new remained to be discovered in this field. Far from this being the case, a most notable impression gained from the speakers at this symposium was the great dearth of reliable relevant information.

The lack of information and the misconception about many aspects of human energy metabolism were mentioned in relation to some of the "Basic Physiological Factors affecting Calorie Balance", the title of the paper by Dr. J. V. G. A. Durnin (University of Glasgow). Part of the complexity of 'calorie balance' arises because of the difficulty of assessing methodological accuracy; there is no sure way of knowing whether the intake of calories or the expenditure of energy represents the 'normal' or usual state for the individual subject. $\mathrm{He}$, or she, may be eating more or less, during the experimental study, than would normally be the case, or the level of energy expenditure may be altered. Since it is extremely difficult under field conditions to carry out such measurements over a period of more than a few days, theoretically one possible way of finding a departure from the normal would be to measure a change of weight in the subject. Part of this paper and a large part of the discussion were devoted to the balance of weight, and Dr. Durnin showed results for young men, weighed under highly stan- dardized conditions, where their gross body-weight changed by up to $3 \mathrm{lb}$. (1.4 kgm.) from day to day. Such changes, presumably almost entirely due to alterations in the fluid content of the body, make the weighing of subjects quite worthless in regard to the balance of energy.

Other factors dealt with were exercise (so often discussed emotionally rather than rationally), climate, age (growth in the young and inversion of growth in the old) and the efficiency of utilization of food by the body.

That most of us do not normally attain balance of energy from day to day was shown clearly by an analysis on the results for 69 individuals, where only 4 subjects had a significant correlation between their intake and output of energy; our 'appetite' is a fickle mechanism.

Calorie balance is probably a long-term control, which may be effected by centres in the hypothalamus responding to changes in the fat depots of the body and then influencing the general level of food intake and perhaps even of bodily activity. In this control, the reticular activating system of the brain stem, the limbic system and the hypothalamus may act together. This was suggested by Dr. G. C. Kennedy (University of Cambridge) in his paper "Central Nervous Regulation of Calorie Balance". The importance of exercise was stressed, both in relation to man and to studies on animals; much work on rats or mice is not comparable because of varying levels of physical activity resulting from restrictions imposed by some types of animal cages. Similarities between a 42 stone (267 kgm.) obese patient and experimentally obese rats were shown by both the astonishing hyperphagia and also lack of resentment or discomfort when food was restricted. They were also alike in their inactivity.

It has been the custom, at certain levels of medical therapeutics, to treat obesity as if it were primarily an endocrine disorder and give the patient mixtures of tissue extracts from various endocrine glands. 
The traditional blame attached, by many clinicians, to the endocrine system in the causation of obesity was repudiated by Dr. Raymond Greene (London) in "The Effects of the Endocrine System on Calorie Balance in Man". The several endocrine glands were considered in brief, abnormalities of some of them being associated with modifications in the water content of the body; occasionally obesity occurs concomitantly with endocrine pathology but rarely, he thought, is hormonal disorder of any great importance. Dr. Greene suggested that a more interesting discourse, rather than the prescribed one, might have been on "the influence of nutrition on the endocrine system".

Within recent years some changes in outlook on the nature of fat metabolism have been apparent. It has been suggested, although not always with convincing experimental evidence, that the fat depots of the body are far from inactive tissues and indeed are continuously being mobilized. Their nerve supply is stated to be extensive, although most histologists appear to have some difficulty in finding many nerve endings, as opposed to nerve fibres, in adipose tissue. Prof. A. Kekwick and his colleagues have been engaged in studying the "Metabolism of Adipose Tissue", the subject of a paper by Dr. T. M. Chalmers (Middlesex Hospital, London). $\mathrm{H}_{\theta}$ suggested that free fatty acid and not glucose might be the principal energy substrate, because of its rapid turnover in the plasma. The fat-mobilizing substance, found in the urine of fasting men, might have some physiological role; its release in the urine apparently depends on carbohydrate metabolism, this being demonstrated in one obese patient treated with equicalorific diets containing varying amounts of carbohydrate.

The second half of the meeting began with a paper on "Energy Expenditure and Calorie Intake in Young Men" by Dr. O. G. Edholm (Medical Research Council, London). In a series of 6 surveys, on 54 Army recruits, he had found variations in energy expenditure of from 2,200 to 6,100 kealories/day and of intake from 671 to $8,750 \mathrm{kcalories} / \mathrm{day}$, with means of about 3,800. The lack of agreement between calorie balance and the weight balance of the body was again stressed, when these were measured over periods of a few days. There is not sufficient information at present to determine the effect, if any, of severe cold on calorie balance. Work carried out in the Antarctic has shown that men put on weight in the winter and lose weight in the summer; this might be due to climatological factors, but equally well might reflect different social circumstances.

The amount and the rationale of the extra "Calorie Requirements in Pregnancy" were discussed by Dr. A. M. Thomson and Dr. F. Hytten (University of Aberdeen). From a series of several hundred healthy primiparæ studied in Aberdeen, it seemed that an average of about $4 \mathrm{kgm}$. of fat was added to the mother's tissues by the time pregnancy ended. This is almost certainly physiological, and is required to help the mother in the needs of lactation ; however, if breast-feeding is becoming much less common, as appears to be true in Western society, this extra gain in weight may predispose to future obesity. The authors emphasized the small amount of experimental information on the exact increase in food taken during pregnancy and the degree of the changes, if any at all, in the amount of physical activity of the woman; this has a considerable bearing on any extra allowance recommended.

From the interesting paper by Dr. E. M. Widdowson (University of Cambridge) on "Energy Balance in Early Life", new-born babies are frequently subjected to considerable stress by deprivation of food. When this happens, the fat stores of the body are quickly used up, particularly if the baby is required to maintain a high metabolic rate. This will be necessary if the environmental temperature does not fall within the critical range $32-35^{\circ} \mathrm{C}$. Babies appear to be as variable in their energy expenditure as adults, and it seems unphysiological to expect all babies to require similar quantities of food.

The discussion of the papers was relevant, interesting and prolonged; the meeting was a most successful one.
J. V. G. A. Durnin

\section{WATER IN AUSTRALIA}

$\mathrm{M}$ AN'S existence depends on an adequate supply of water, and in Australia, as in few other countries, future development will depend on using water resources to the full. An important step in the utilization of water is the understanding of its properties, and the Victorian Branch of the Royal Australian Chemical Institute found no difficulty in attracting some fifty sciontists from four Australian States, whose interests ranged from engineering to biology, to a three-day discussion on this broad topic. The meeting was hold at Warburton, Victoria, in late November.

In the opening talk Dr. J. A. Barker reviewod the structure of hydrates and of ice as an introduction to the loosely packed structure of liquid water in which the molecules are prevented from coming into closest proximity by tetrahedrally arranged hydrogen bonds. Each pattern of hydrogen bonds persists for periods of the order of $10^{-12}$ sec. The theory of Pople, based on this picture, would require each water molecule to have 4 nearest neighbours, 12 next nearest and 36 third nearest, whereas the correspond- ing figures obtained from analysis of $\mathrm{X}$-ray diffraction data are 4, 11 and 22 . Dr. Barker contrasted the anomalous properties of water that result from this structure-the decrease in solubility of gases with increasing temperature, the high negative temperature coefficient of viscosity - with the properties of more normal liquids in which the molecules are closely packed.

Evidence consistent with this structure was presented by Dr. S. D. Hamann, who described some of the effects of pressure on the proporties of water and showed how many of the anomalies disappear as the moloculos become close-packed at pressures greator than 2,000 atm. Thus with increasing pressure the thermal expansion of water became 'normal', its sound absorption decreased as pressure broke down its structure, and its dielectric constant decreased. Measurements of the conductivity of water under pressures up to $130,000 \mathrm{~atm}$. had produced increases in ionization so that its conductivity equals that of a $5-N$ solution of hydrochloric acid. Pressures of this order are probably reached at depths of $400 \mathrm{~km}$. in 